\title{
ON PERIODIC SOLUTIONS OF SUPERLINEAR PARABOLIC PROBLEMS
}

\author{
BY
}

M. J. ESTEBAN

\begin{abstract}
In this paper we study the existence of positive nontrivial periodic solutions of semilinear parabolic problems. Most of the nonlinearities considered are of the superlinear type. Some bifurcation results are proved as well.
\end{abstract}

Introduction. The goal of this paper is to give some existence results of positive periodic solutions of parabolic semilinear problems:

$$
\left\{\begin{array}{l}
u_{t}-L u=f(t, x, u) \text { in }(0, T) \times \Omega, \\
u=0 \text { on }(0, T) \times \partial \Omega, \\
u(0)=u(T) \text { in } \bar{\Omega} ; \quad u>0 \text { in }(0, T) \times \Omega,
\end{array}\right.
$$

where $\Omega$ is a bounded regular domain of $\mathbf{R}^{N}, \partial \Omega$ is its boundary, and $L$ is a uniformly elliptic operator.

We will specially treat two cases:

(A) $f(t, x, s)=0$ when $s=0$ and $f(t, x, \cdot)$ grows superlinearly at $+\infty$.

(B) $f(t, x, 0)>0$ for all $(t, x) \in[0, T] \times \bar{\Omega}$.

In some particular cases we will take $f(t, x, s)$ convex in $s$, for $s \geqslant 0$.

Let us give two examples of our main results:

(a) We suppose that $f(t, x, s)=m(t) s^{\alpha}$, and $m \in W^{1, \infty}(0, T), m>0$. Then we prove that under suitable assumptions on $m$, there exists a solution of (1) for $1<\alpha<(3 N+8) /(3 N-4)$.

(b) Secondly we prove that if $f$ depends also on a real positive parameter $\lambda$, and has the form $f(t, x, s)=\lambda l(t, x, s)+h(t, x)$, where $l$ is positive and $h$ is nonnegative, then there exists a branch of solutions of (1),

$$
\mathscr{A}=\{(\lambda, u(\lambda)) \mid, 0 \leqslant \lambda<\underline{\lambda}<+\infty\} .
$$

In several cases we can also prove that

(i) there is a solution $\underline{u}$ for $\lambda=\underline{\lambda}$,

(ii) $(\underline{\lambda}, \underline{u})$ is a bending point for $\mathscr{A}$,

(iii) there exist at least two solutions of (1) for $0<\lambda<\underline{\lambda}$.

Received by the editors October 1, 1984.

1980 Mathematics Subject Classification. Primary 35K55, 35B10.

Key words and phrases. Superlinear parabolic problems, periodic solutions, positive solutions, a priori estimates. 
The types of results (a) are obtained in two steps:

- we first prove that the solutions (1) are uniformly bounded in $L^{\infty}((0, T) \times \Omega)$;

- then we apply a topological degree argument to infer the existence of at least one solution of (1).

In case (b) topological degree theory is used together with a priori estimates and the implicit function theorem.

In both cases the a priori $L^{\infty}$-estimates are obtained by one of these techniques:

(1) First we get $L^{1}\left(Q_{T}\right)$-estimates for $u$ and $f(u)$, and then an $L^{2}\left(Q_{T}\right)$-estimate for $\nabla u$. In some situations this is enough to infer the $L^{\infty}$-estimate by a boot-strap argument. Otherwise, intermediate estimates in some Lorentz espaces will be used. This technique is strongly based upon some arguments used in $[8,10]$.

(2) The second method consists in assuming that the $L^{\infty}$-estimates do not hold, and then reaching a contradiction via the study of some associated problems in $\mathbf{R}^{N+1}$ or some half-espace of it. This technique was used in [12] for the elliptic problem.

Previous work on this subject has been done by J. S. Kolesov [14], H. Amann [1, 2], A. C. Lazer [16], P. Hess [13] and H. Brézis-L. Nirenberg [6]. Kolesov proves that the method of sub- and supersolutions works also in the parabolic-periodic case. $\mathrm{H}$. Brézis, L. Nirenberg, A. C. Lazer, A. Beltramo-P. Hess [4] and P. Hess use Kolesov's result to prove the existence of periodic solutions of parabolic problems. Roughly speaking, these authors deal with the sublinear situation, that is, when $f(t, x, \cdot)$ does not grow too quickly at $+\infty$.

On the other hand, the results of $\mathrm{H}$. Amann rely on the existence of general invariant sets for the parabolic-periodic problem. Finally, A. Castro, A. C. Lazer, and A. Beltramo-P. Hess study the existence and properties of a principal eigenvalue for parabolic-periodic problems. Then they obtain some existence results in the sublinear case.

This paper is organized as follows:

In §I we give some notation and definitions. We also explain shortly what kinds of results are known about the existence of a principal eigenvalue.

§II is devoted to the statement of the main results obtained in case (A). In particular, we will consider that $f$ has the form $f(t, x, s)=m(t) g(s)$, with $g(s)=s^{\alpha}$. Then we will assume for simplicity that $f$ does not depend on $x$ : dependence on $x$ can be treated in the same way after a suitable change in the assumptions.

In §III we give the proofs of the results stated in §II. In §IV we state the theorems concerning the general case (A), that is, when $f(t, x, s)=m(t) g(s)$, and $g(s)$ is not necessarily a power. We will omit the proofs of these theorems, since they are quite similar to those of $\S I I I$.

Finally, in $\S \mathrm{V}$ we will consider case (B), and we will explicit the behavior of the solution set with respect to a positive parameter $\lambda$.

I. Notation and definitions. As said in the Introduction, $\Omega$ is a bounded regular domain of $\mathbf{R}^{N}$, of boundary $\partial \Omega$. In all that follows we denote by $Q_{T}$ the open set $(0, T) \times \Omega$, and by $S_{T}$ its lateral boundary, i.e., $S_{T}=(0, T) \times \partial \Omega$. 
The functional spaces which appear in this paper are Sobolev and Hölder spaces:

For $r, s \in \mathbf{N}, p \in \mathbf{R}$, we define

$$
\begin{aligned}
& W^{r, p}(\Omega)=\left\{u: \Omega \rightarrow \mathbf{R} \mid D_{x}^{i} u \in L^{p}(\Omega), i \leqslant r\right\}, \\
& W^{r, s, p}\left(Q_{T}\right)=\left\{u: Q_{T} \rightarrow \mathbf{R} \mid D_{x}^{i} u, D_{t}^{\prime} u \in L^{p}(\Omega), i \leqslant r, l \leqslant s\right\} .
\end{aligned}
$$

For $\mu \in \mathbf{R}^{+}, 0<\mu<1$ and $\alpha, \beta \in N$,

$$
\begin{aligned}
& C^{\mu, \mu / 2}\left(Q_{T}\right)=\left\{u \in C\left(Q_{T}\right) \mid \sup _{\substack{\left(t_{k}, x_{k}\right) \in Q_{T} \\
\left(t_{1}, x_{1}\right) \neq\left(t_{2}, x_{2}\right)}} \frac{\left|u\left(t_{1}, x_{1}\right)-u\left(t_{2}, x_{2}\right)\right|}{\left|x_{1}-x_{2}\right|^{\mu}+\left|t_{1}-t_{2}\right|^{\mu / 2}}<+\infty\right\}, \\
& C^{\alpha+\mu, \beta+\mu / 2}\left(Q_{T}\right)=\left\{u \mid D_{x}^{i} u, D_{t}^{l} u \in C^{\mu, \mu / 2}\left(Q_{T}\right), i \leqslant \alpha, l \leqslant \beta\right\} .
\end{aligned}
$$

On the other hand, the derivatives of $u$ with respect to $t$ will be denoted by $u_{t}$, $u_{t t}$, etc., and with respect to $x$ by $u_{i_{1} \cdots i_{m}}=\partial^{m} u /\left(\partial x_{1} \cdots \partial x_{m}\right) ; \Delta u=\sum_{i=1}^{N} \mu_{i i}$ and $\nabla u=\left(u_{1} \cdots u_{N}\right)$ are the usual notations for the Laplacian and the gradient operators.

Next let us state what is known about the existence of a principle eigenvalue for periodic-parabolic problems.

A. Beltramo and P. Hess (see [4]) showed that if they consider the linear eigenvalue problem

$$
\left\{\begin{array}{l}
\mathbf{L} u=\lambda m(t, x) u \text { in } \mathbf{R} \times \Omega, \\
u=0 \text { on } \mathbf{R} \times \partial \Omega, \\
u(0, \cdot)=u(T, \cdot) \text { in } \bar{\Omega},
\end{array}\right.
$$

where $\mathbf{L}$ is a uniform parabolic operator, the following holds:

Theorem 1 (A. Beltramo AND P. Hess [4]). If there exists $x_{0} \in \Omega$ such that $\int_{0}^{T} m\left(t, x_{0}\right) d t>0$, then (2) has a unique positive eigenvalue $\lambda_{1}(m)$ having a positive eigenfunction $u=u_{1}(m)$ and

(i) if $\lambda$ is an eigenvalue of (2), $\lambda \geqslant \lambda_{1}(m)$,

(ii) $\lambda_{1}(m)$ is algebraically simple,

(iii) Assume that $0 \leqslant \lambda<\lambda_{1}(m)$, then for all $h \in C^{\mu, \mu / 2}\left(Q_{T}\right)$ there exists a unique solution of

$$
\left\{\begin{array}{l}
\mathbf{L} u=\lambda m(t, x) u+h \text { in } Q_{T} \\
u=0 \text { on } S_{T}, \\
u(0)=u(T) \text { in } \bar{\Omega} .
\end{array}\right.
$$

Moreover, $h \geqslant 0$ implies that $u \geqslant 0$.

REMARK 2 . The case $m \equiv 1$ had been previously studied by A. Castro and A. C. Lazer (see [7]).

REMARK 3. Theorem 1 points out the similarities of the elliptic and the periodicparabolic cases. 
Finally, throughout this paper $C$ will denote various positive constants independent of $u$, and $r^{+}$(resp. $r^{-}$) will denote the positive (resp. negative) part of the real number $r$.

II. Main results in case (A). Throughout this section, as well as in the next two, we consider that $f$ has the form $f(t, x, s)=m(t) g(s)$. For commodity we take $g$ independent of $x$. On the other hand, the separation of variables makes the assumptions clearer.

Let us first begin with $g(s)=s^{\alpha}$. Under this form the results will appear more clearly and the general case (A) will be treated in §IV.

Thus we are now interested in the existence of solutions of

$$
\left\{\begin{array}{l}
u_{t}-\Delta u=m(t) u^{\alpha} \quad \text { in } Q_{T} \\
u=0 \text { on } S_{T} ; \quad u>0 \text { in } Q_{T} \\
u(0)=u(T) \text { in } \bar{\Omega}
\end{array}\right.
$$

where we assume that

$$
\begin{gathered}
m \in L^{\infty}(0, T), \\
\inf _{0 \leqslant t \leqslant T} m(t)=m_{0}>0 .
\end{gathered}
$$

Now we can state our first result concerning (4), or the parabolic-periodic problem which appears when we replace $-\Delta$ by a general elliptic operator $L$.

THEOREM 4. Assume (5)-(6). Then if $1<\alpha<(N+2) / N$, (4) has at least one solution in $C^{2+\mu, 1+\mu / 2}\left(Q_{T}\right)$, for $0<\mu<1$. Moreover, in this case we can allow in (4) any elliptic operator $L$ and not necessarily $-\Delta$.

REMARK 5. If we are interested in the range of real numbers $\alpha$ for which (4) has at least a solution, this theorem yields a very restrictive answer. On the other hand, $m$ is allowed to be more general than in the other results below.

TheORem 6. Assume that $m$ satisfies (6) and

$$
\begin{gathered}
m \in W^{1, \infty}(0, T), \\
m(0)=m(T) .
\end{gathered}
$$

Then if $1<\alpha<(N+1) /(N-1)$, (4) has at least one solution in $C^{2+\mu, 1+\mu / 2}\left(Q_{T}\right)$ for $0<\mu<1$.

Theorem 7 provides the best range for $\alpha$ to our knowledge. However, more restrictive assumptions will be made on $m$.

Let us define the following positive real number (depending on $\Omega$ ):

$$
R(\Omega)=\inf _{x \in \mathbf{R}^{N}}\left(\sup _{y \in \Omega}|x-y|-\inf _{y \in \Omega}|x-y|\right) .
$$

Then we assume that besides (6)-(8) $m$ satisfies

$$
\sup _{0 \leqslant t \leqslant T} \frac{\left(m^{\prime}(t)\right)^{-}}{m(t)}<\frac{4 N-2(N-2)(\alpha+1)}{R(\Omega)} .
$$


Then we can state the following

THEOREM 7. Under the above assumptions, and if $\alpha$ satisfies

$$
1<\alpha<(3 N+8) /(3 N-4),
$$

then (4) has at least one solution in $C^{2+\mu, 1+\mu / 2}\left(Q_{T}\right)$ for $0<\mu<1$.

REMARK 8. As Theorem 7 shows, $(3 N+8) /(3 N-4)$ seems to be, up to now, the best upper bound for $\alpha$. At this moment we are not able to prove nonexistence results for (4) when $\alpha \geqslant \alpha^{*}$, for some $\alpha^{*} \geqslant(3 N+8) /(3 N-4)$. Indeed, such a result would be very useful in order to set a limit for the admissible growth of the nonlinearity $g(s)$ when $s$ goes to $+\infty$. Clearly $\alpha$ has to be less than $(N+2) /(N-2)$. Indeed, if we take $m(t) \equiv m_{0}$, all the solutions of (4) are independent of $t$ and (4) becomes an elliptic problem. Then, Pohozaev's identity (see [21]) shows that in general $\alpha<(N+2) /(N-2)$.

III. Proofs of Theorems 5-7. The proofs of these theorems will be divided into several steps. We first obtain a priori $L^{\infty}\left(Q_{T}\right)$-estimates for the solutions of (4). Then the existence will follow by a topological degree argument. In the case of Theorem 4 we will use two different methods to obtain the a priori estimates. One of them will allow us to consider in (4) general parabolic operators of the form $(\partial / \partial t)-L$, where $L$ is a uniformly elliptic operator.

The a priori estimates are obtained by two different techniques introduced for the elliptic case-one by D. G. de Figueiredo, P. L. Lions and R. D. Nussbaum in [10], and the other one by B. Gidas and J. Spruck in [12].

Proposition 9. Assume that $m$ satisfies (5)-(6) and that $\alpha>1$. Then all the classical solutions of (4) are uniformly bounded in $L^{\alpha}\left(Q_{T}\right)$.

Proof. First we see that if $u$ is a solution of $(4),\|u\|_{L_{\text {loc }}^{\alpha}\left(Q_{T}\right)} \leqslant C$, with $C$ independent of $u$. Indeed, we multiply equation (4) by $v_{1}$, a positive eigenfunction associated with $\lambda_{1}(m)^{1}$ and we find

$$
\iint_{Q_{T}} m(t) u^{\alpha} v_{1} d t d x=\lambda_{1}(m) \iint_{Q_{T}} u v_{1} d t d x
$$

We immediately infer the existence of a constant $C>0$ such that

$$
\iint_{Q_{T}} u v_{1} d t d x \leqslant C, \text { and therefore, } \iint_{Q_{T}} u^{\alpha} v_{1} d t d x \leqslant C .
$$

Next, if we know that $u$ is uniformly bounded in $L^{\infty}$ of a fixed neighborhood of $S_{T}$, we have

$$
\iint_{Q_{T}} u^{\alpha} d t d x \leqslant C
$$

Let us then complete the proof of Proposition 9 with the following

LEMma 10. If $m$ satisfies (5)-(6) and $\alpha>1$, then there exists a neighborhood of $S_{T}$ (independent of $u$ ) where $u$ and $\nabla u$ are uniformly bounded in $L^{\infty}$-norm.

\footnotetext{
${ }^{1}$ Relatively to the adjoint operator of $\partial_{t}-\Delta,-\partial_{t}-\Delta$.
} 
Proof. This proof is quite similar to the one made in the elliptic case by B. Gidas, W.-M. Ni and L. Nirenberg in [11]. We will then be quite brief. Let us introduce some notation:

If $\Omega$ is a bounded, convex domain of $\mathbf{R}^{N}$ and $\gamma \in \mathbf{R}^{N}-\{0\}$, we define $\Sigma(\lambda, \gamma)=$ $\{x \in \Omega \mid x \cdot \gamma<0\}$, and $\Sigma^{\prime}(\lambda, \gamma)$ will be the reflection of $\Sigma(\lambda, \gamma)$ across the hyperplane $T_{\lambda}=\left\{x \in \mathbf{R}^{N} \mid x \cdot \gamma=0\right\}$,

We fix $x_{0} \in \partial \Omega$. Then there exists a $\lambda_{0}$ such that $T_{\lambda_{0}} \cap \partial \Omega=\left\{x_{0}\right\}$. Moreover, as $\lambda$ increases (starting at $\left.\lambda_{0}\right)$, it reaches a value $\gamma_{1}$ such that either $\Sigma\left(\lambda_{1}, \gamma\right)$ is internally tangent to $\partial \Omega$ or $T_{\gamma_{1}}$ intersects $\partial \Omega$ somewhere tangentially.

Then under our assumptions we can prove that

$$
\left\{\begin{array}{l}
\text { for all } \lambda \in\left(\lambda_{0}, \lambda_{1}\right), \text { for all }(t, x) \in[0, T] \times \Sigma(\lambda, \gamma), \\
u(t, x)<u\left(t, x^{\lambda}\right) \text { and } \nabla_{x} u(t, x) \cdot \gamma<0 \\
\text { where } x^{\lambda} \text { denotes the reflection of } x \text { across } T_{\lambda} .
\end{array}\right.
$$

This result is stated (without proof) in [11, Theorem 5.2] in the case of a parabolic problem with initial value satisfying $u(0, x)<u\left(0, x^{\lambda}\right)$ for all $x \in \Sigma(\lambda, \gamma)$, for all $\lambda \in\left(\lambda_{0}, \gamma_{1}\right)$. The proof in the periodic-parabolic case is almost the same. Indeed, these types of results rely strongly on the maximum principle. In the periodic case the assumption on the initial value is skipped because $u(0)=u(T)$, and then in that case the maximum principle gives a more complete information about the solution.

Next we prove that (13) actually implies the existence of two positive constants $r_{0}$, $\beta$, such that $u(t, x-r \nu)$ is nondecreasing in $r \in\left[0, r_{0}\right]$ for all $(t, x) \in \bar{S}_{T}$ and for all $\nu \in \mathbf{R}^{N}$ with $|\nu|=1$ and $\nu \cdot n(x) \geqslant \beta$, where $\eta(x)$ denotes the outward unitary normal vector to $\partial \Omega$ at $x$.

The boundedness of $u$ in a fixed neighborhood of $S_{T}$ follows from the combination of this last result with (12).

We will prove next that $u^{\alpha}$ is uniformly bounded in some space better than $L^{1}\left(Q_{T}\right)$ :

Proposition 11. Under assumptions (5)-(6) and if $1<\alpha<(N+2) / N$, we can find two positive constants, $C, \delta$, such that $\|u\|_{L^{\alpha+\delta}\left(Q_{T}\right)} \leqslant C$.

Proof. Since by Proposition $9, u^{\alpha}$ is bounded in $L^{1}\left(Q_{T}\right)$, we see that $u(t, x)$ can be written as

$$
u(t, x)=K_{\Omega}(t, x, \tau, \xi) *\left(m(\tau) u^{\alpha}(\tau, \xi)\right),
$$

where $K_{\Omega}$ is the Green's function for $\mathbf{L}=(\partial / \partial t)-\Delta$ in $Q_{T}$, with periodic conditions. We know also (see [3]) that for all $q \in\left[1,(N+2) / N\right.$ ) there exists $C_{q}>0$ such that

$$
\|K(t, x ; \cdot, \cdot)\|_{L^{q}\left(Q_{T}\right)} \leqslant C_{q} \quad \text { for all }(t, x) \in Q_{T} .
$$

Therefore $u$ is uniformly bounded in $L^{q}\left(Q_{T}\right)$ for $1 \leqslant q<(N+2) / N$, and $u^{\alpha}$ will be bounded in $L^{1+\varepsilon}\left(Q_{T}\right)$ for some $\varepsilon>0$.

We finally prove in the next proposition the existence of $L^{\infty}\left(Q_{T}\right)$-estimates for the solutions of (4). 
Proposition 12. If $m$ satisfies (5)-(6) and $1<\alpha<(N+2) / N$, then all solutions of (4) are uniformly bounded in $L^{\infty}\left(Q_{T}\right)$.

Proof. Standard regularity results show that if $u$ is a solution of (4) and $u^{\alpha}$ is in $L^{p}\left(Q_{T}\right)$ with $p>1$, then $u \in W^{2,1, p}\left(Q_{T}\right)$ and

$$
\|u\|_{W^{2,1, p}\left(Q_{T}\right)} \leqslant C\left\|u^{\alpha}\right\|_{L^{p}\left(Q_{T}\right)} .
$$

Moreover, by Sobolev's embedding theorem, we know that $W^{2,1, p}\left(Q_{T}\right)$ is continuously embedded in $L^{q}\left(Q_{T}\right)$ for $1 \leqslant q \leqslant(N+2) p /(N+2-2 p)$. Then, since $\alpha<(N+2) / N$, we see that $u^{\alpha}$ is bounded in $L^{p_{2}}\left(Q_{T}\right)$ with $p_{2}>p$. The $L^{\infty}$ estimate will follow from the iteration of this argument.

Before ending the proof of Theorem 4 let us give another (and more direct) proof of Proposition 12. The advantage of the proof given above is that it will be used in the proofs of Theorems 6 and 7. On the other hand, the new proof below can be used when the elliptic operator involved in (4) is a general one, and not necessarily $-\Delta$. This is then necessary in order to give a complete proof of both Proposition 12 and Theorem 4.

Alternative proof of Proposition 12. This proof is done by using a technique introduced by B. Gidas and J. Spruck (see [12]) to obtain a priori $L^{\infty}$-estimates for the solutions of superlinear elliptic problems. The ideas are almost the same. Then we will only sketch the proof.

We take for simplicity (4) and not the more general parabolic problem where $(-\Delta)$ is replaced by a general elliptic operator $L$.

We assume by contradiction that the regular solutions of (4) are not uniformly bounded in $L^{\infty}\left(Q_{T}\right)$. Therefore there exists an unbounded sequence of real numbers, $\left\{M_{k}\right\}$, and a sequence of solutions of (4), $\left\{u_{k}\right\}$, such that $\left\|u_{k}\right\|_{L^{\infty}\left(Q_{T}\right)}=u_{k}\left(\bar{t}_{k}, P_{k}\right)$ $=M_{k}$. For every $k$ let us define $\lambda_{k}, y, \tau$ and $v_{k}$ as follows:

$$
\lambda_{k}^{2 /(\alpha-1)} M_{k}=1, \quad y=\frac{x-P_{k}}{\lambda_{k}}, \quad \tau=\frac{t-\bar{t}_{k}}{\lambda_{k}^{2}} \quad \text { and } \quad v_{k}(\tau, y)=\lambda_{k}^{2 /(\alpha-1)} u_{k}(t, x) \text {. }
$$

Then, it is obvious that, for every $k,\left\|v_{k}\right\|_{L^{\infty}}=v_{k}(0,0)=1$. We now consider two different situations:

First case: There exists an accumulation point of $\left\{\left(\bar{t}_{k}, P_{k}\right)\right\},(\bar{t}, P)$ with $(\bar{t}, P) \in Q_{T}$.

In this case there exists a $\delta>0$ such that the functions $v_{k}$ are well defined in a set $A_{\delta}^{k}=\left(-\delta / \lambda_{k}^{2}, \delta / \lambda_{k}^{2}\right) \times B_{\delta / \lambda_{k}}(0)$, where $B_{\beta}(M)$ denotes the ball of $\mathbf{R}^{N}$ of radius $\beta$ centered at $M$.

We easily see that $v_{k}$ satisfies the following equation in $A_{\delta}^{k}$ :

$$
\left(v_{k}\right)_{t}-\Delta v_{k}=m(\tau) v_{k}^{\alpha}
$$

Since $v_{k}$ is uniformly bounded by 1 , standard regularity results yield the convergence of $\left\{v_{k}\right\}$ in $C^{2+\mu, 1+\mu / 2}\left(B_{R}(0)\right)$ to a certain $v^{R} \in C^{2,1}\left(\mathbf{R} \times \mathbf{R}^{N}\right)$ for any $R>0$. Repeating this argument for $R^{\prime}>R$ we see that $v^{R^{\prime}} / B_{R}(0)=v^{R}$. This means that 
$\left\{v_{k}\right\}$ converges locally to a function $v \in C^{2,1}\left(\mathbf{R} \times \mathbf{R}^{N}\right)$ which satisfies

$$
\left\{\begin{array}{l}
v_{t}-\Delta v=c v^{\alpha} \quad \text { in } \mathbf{R} \times \mathbf{R}^{N} \\
v>0 \quad \text { in } \mathbf{R} \times \mathbf{R}^{N} \\
\|v\|_{L^{\infty}}=v(0,0)=1
\end{array}\right.
$$

and by means of a change of variable we can consider that $C=1$.

The proof will then be finished with the following nonexistence result:

Proposition 13. If $u \in C^{2,1}\left(\mathbf{R} \times \mathbf{R}^{N}\right)$ is a solution of

$$
\left\{\begin{array}{l}
u_{t}-\Delta u=u^{\alpha} \quad \text { in } \mathbf{R} \times \mathbf{R}^{N}, \\
u \geqslant 0 ; \quad u \in L^{\infty}\left(\mathbf{R} \times \mathbf{R}^{N}\right),
\end{array}\right.
$$

with $1<\alpha<(N+2) / N$, then $u \equiv 0$.

PROOF. If $B_{1}$ is the unit ball of $\mathbf{R}^{N}$ centered at 0 , let $v_{1}$ be a positive eigenfunction of $(-\Delta)$ when it acts on $H_{0}^{1}\left(B_{1}\right)$ such that $\left\|v_{1}\right\|_{L^{1}\left(B_{1}\right)}=1$, and let $\lambda_{1}$ be the corresponding eigenvalue.

Then for every $R \geqslant 1$ we define in $B_{R}$ the function $v_{R}(x)=\left(1 / R^{N}\right) v_{1}(x / R)$. It is obvious that $v_{R}$ is a positive eigenfunction of $(-\Delta)$ when acting on $H_{0}^{1}\left(B_{R}\right)$, that $\left\|v_{R}\right\|_{L^{1}\left(B_{R}\right)}=1$ and that the corresponding eigenvalue is $\lambda_{1} / R^{2}$.

We multiply (14) by $v_{R}$ and after some integration by parts we find

$$
\int_{B_{R}} u_{t} v_{R} d x+\frac{\lambda_{1}}{R^{2}} \int_{B_{R}} u v_{R} d s \geqslant \int_{B_{R}} u^{\alpha} v_{R} d x
$$

But since $\left\|v_{R}\right\|_{L^{1}\left(B_{R}\right)}=1$

$$
\int_{B_{R}} u^{\alpha} v_{R} d x \geqslant\left(\int_{B_{R}} u v_{R} d x\right)^{\alpha} .
$$

If we then define $f(t)=\int_{B_{R}} u(t) v_{R} d x$, we have $f^{\prime}(t) \geqslant f^{\alpha}(t)-\left(\lambda_{1} / R^{2}\right) f(t)$.

Since $f$ is bounded and positive, it follows from the study of the real function $\left(x^{\alpha}-\left(\lambda_{1} / R^{2}\right) x\right)$ that $f(t) \leqslant \lambda_{1}^{1(\alpha-1)} / R^{2 /(\alpha-1)}$. Indeed, if for some $t_{0}, f\left(t_{0}\right)>$ $\lambda_{1} / R^{2 /(\alpha-1)}$, then for all $t \geqslant t_{0}, f^{\prime}(t) \geqslant f\left(t_{0}\right)^{\alpha}-\left(\lambda_{1} / R^{2}\right) f\left(t_{0}\right)>0$, and this is impossible.

Then

$$
\int_{B_{R}} u v_{R} d x \leqslant \frac{\lambda_{1}^{1 /(\alpha-1)}}{R^{2 /(\alpha-1)}} \quad \forall t, \forall R \geqslant 1 .
$$

Moreover

$$
\int_{B_{R}} u v_{R} d x \geqslant \int_{B_{R / 2}} u v_{R} d x \geqslant \frac{v_{1}\left(\frac{1}{2}\right)}{R^{N}} \int_{B_{R / 2}} u d x
$$


Therefore

$$
\int_{B_{R / 2}} u d x \leqslant \frac{C}{R^{2 /(\alpha-1)-N}}, \quad \forall R \geqslant 1 .
$$

If $\alpha<(N+2) / N, 2 /(\alpha-1)-N>0$, and this contradiction ends the proof.

Second case: All the accumulation points of $\left\{\left(\bar{t}_{k}, P_{k}\right)\right\}$ lie on $S_{T}$.

REMARK. The third possible case, that is, when all accumulation points lie in $\{0$, $T\} \times \bar{\Omega}$ reduces to the first one, because we can always define $u$ in $(-T, 2 T) \times \Omega$ by periodicity and then do as we did in the first case.

First we suppose for simplicity that if $(\bar{t}, P) \in S_{T}$ is an accumulation point of $\left\{\left(\bar{t}_{k}, P_{k}\right)\right\}$, near $P, \partial \Omega$ lies on $\left\{x_{N}=0\right\}$ and the $n$th coordinate of $P_{k}$ is positive.

Then we make exactly the same changes of variables and function as before and we see that in this case $v^{k}$ is well defined in a set:

$$
D_{\delta}^{k}=\left(-\frac{\delta}{\lambda_{k}^{2}}, \frac{\delta}{\lambda_{k}^{2}}\right) \times\left(B_{\delta / \lambda_{k}} \cap\left\{y \mid y_{N}>-\frac{d_{k}}{\lambda_{k}}\right\}\right),
$$

where $d_{k}=\operatorname{distance}\left(P_{k}, \partial \Omega\right)$, and $y_{N}$ is the $N$ th coordinate of $y$.

Since we have nice estimates again on $v^{k}$, we will prove as before the local convergence of $\left\{v^{k}\right\}$ to a function $v$ which satisfies

$$
\left\{\begin{array}{l}
v_{t}-\Delta v=c v^{\alpha} \quad \text { in } \mathbf{R} \times\left\{y \mid y_{N}>-s\right\}, \\
v=0 \text { on } \mathbf{R} \times\left\{y \mid y_{N}=-s\right\}, \\
v(0)=1,
\end{array}\right.
$$

where $s=\lim _{n \rightarrow+\infty}\left(d_{k} / \lambda_{k}\right)$. As before we can also take $C=1$. Moreover we observe that $s>0$. Indeed, for every $k$, we have

$$
\left|v^{k}(0,0)-v^{k}\left(0, d_{k} / \lambda_{k}\right)\right|=|1-0| \leqslant C d_{k} / \lambda_{k},
$$

where $C$ does not depend on $k$.

If $s=+\infty$, we finish the proof as in the first case. If $0<s<+\infty$, we can extend $v$ to all $\mathbf{R} \times \mathbf{R}^{N}$ as follows:

$$
\tilde{v}\left(\tau, y^{\prime}, y_{N}\right)=\left\{\begin{array}{l}
v\left(\tau, y^{\prime}, y_{N}\right) \quad \text { if } y_{N} \geqslant-s, \\
v\left(\tau, y^{\prime},-2 s-y_{N}\right) \text { if } y_{N}<-s .
\end{array}\right.
$$

Then $v$ will be a solution of (14). Indeed,

$$
\begin{aligned}
\left(\partial v / \partial y_{N}\right)\left(\tau, y^{\prime},-s\right) & =0 \quad \text { for all } \tau, y^{\prime}: \\
\frac{\partial v}{\partial y_{N}}\left(\tau, y^{\prime},-s\right) & =\lim _{k \rightarrow+\infty} \frac{\partial v^{k}}{\partial y_{N}}\left(\tau, y^{\prime},-\frac{d_{k}}{\lambda_{k}}\right), \quad \text { and } \\
\frac{\partial v^{k}}{\partial y_{N}}\left(\tau, y^{\prime},-\frac{d_{k}}{\lambda_{k}}\right) & =\lambda_{k}^{(\alpha+1) /(\alpha-1)} \frac{\partial u^{k}}{\partial x_{N}}\left(t, Q_{k}\right) \quad \text { for some } Q_{k} \in \partial \Omega .
\end{aligned}
$$

Then,

$$
\left|\frac{\partial v^{k}}{\partial y_{N}}\left(\tau, y^{\prime},-\frac{d_{k}}{\lambda_{k}}\right)\right| \leqslant \lambda_{k}^{(\alpha+1) /(\alpha-1)}\|\nabla u\|_{L^{\infty}\left(S_{T}\right)} .
$$


Since by Lemma 10, $\|\nabla u\|_{L^{\infty}\left(S_{T}\right)}$ is bounded independently of $u$, and $\lambda_{k} \rightarrow 0$ as $k \rightarrow+\infty, \tilde{v}$ is well defined and is a solution of (14). Then, by Proposition 13, $\tilde{v} \equiv 0$. This contradiction ends the proof.

Proof of TheOREM 4. Once we have proved the $C^{\infty}$-estimates for the solutions of (4), we will prove the existence of at least one solution by the following topological degree argument:

First we observe that there exists a $\rho>0$ such that if $u$ is a solution of

$$
\left\{\begin{array}{l}
u_{t}-\Delta u=\lambda m(t) u^{\alpha} \quad \text { in } Q_{T}, \\
u=0 \text { on } S_{T} ; \quad u>0 \quad \text { in } Q_{T}, \\
u(0)=u(T) \quad \text { in } \bar{\Omega} ; \quad \lambda \in[0,1]
\end{array}\right.
$$

then $\|u\|_{L^{\infty}\left(Q_{T}\right)}>\rho$ and $\rho$ depends only on $\alpha$.

Indeed, assume that there is a sequence of solutions of (15), $\left\{u_{n}\right\}$, such that $\left\|u_{n}\right\|_{L^{\infty}\left(Q_{T}\right)}$ goes to 0 as $k$ goes to $+\infty$. Then we define $w_{n}=u_{n}\left\|u_{n}\right\|_{L^{\infty}\left(Q_{T}\right)}^{-1}$ and we find

$$
\left\{\begin{array}{l}
\left(w_{n}\right)_{t}-\Delta w_{n}=\lambda u_{n}^{\alpha-1} w_{n} \text { in } Q_{T}, \\
w_{n}=0 \text { on } S_{T} ; \quad w_{n}>0 \text { in } Q_{T}, \\
w_{n}(0)=w_{n}(T) \text { in } \bar{\Omega} .
\end{array}\right.
$$

By our hypothesis, there will be an $n_{0}$ such that $\lambda\left\|u_{n_{0}}\right\|_{L^{\infty}\left(Q_{T}\right)}^{\alpha-1}<\lambda_{1}(m)$. But, because of Theorem 1, this is impossible. This contradiction proves our assertion.

Let us now define the mapping $K_{\lambda}: W^{1, q}\left(Q_{T}\right) \rightarrow W^{1, q}\left(Q_{T}\right)$ by $K_{\lambda} u=v$ if

$$
\left\{\begin{array}{l}
v_{t}-\Delta v=\lambda m(t) u^{\alpha} \quad \text { in } Q_{T} \\
v=0 \quad \text { on } S_{T} ; \quad v>0 \text { in } Q_{T} \\
v(0)=v(T) \text { in } \bar{\Omega}
\end{array}\right.
$$

Whenever $q>N+1, K_{\lambda}$ is well defined and compact, as one easily checks. Moreover for $q>N+1, W^{1, q}\left(Q_{T}\right)$ is continuously embedded in $C^{0}\left(Q_{T}\right)$. Let $C_{q}$ be the best constant of that embedding, and $\rho^{\prime}=\rho / C_{q}$. Then if $d\left(I-K_{\lambda}, B_{\rho^{\prime}}, 0\right)$ denotes the Leray-Schauder topological degree of $I-K_{\lambda}$ in $B_{\rho^{\prime}}$ with respect to 0 (see [17, 21], this number does not depend on $\lambda$ for $\lambda \in[0,1]$ ). Indeed, since $K_{\lambda}$ has no fixed points on $\partial B_{\rho^{\prime}}$, the homotopy invariance property of the degree applies. On the other hand, $K_{0}=0$. Hence, $d\left(I-K_{1}, B_{\rho^{\prime}}, 0\right)=1$.

Let us now introduce a new family of operators from $W^{1, q}\left(Q_{T}\right)$ into itself: We say that $T_{L} u=v$ if

$$
\left\{\begin{array}{l}
v_{t}-\Delta v=m(t)\left(u^{\alpha}+L u+L\right) \text { in } Q_{T} \\
v=0 \text { on } S_{T} ; \quad v>0 \text { in } Q_{T} \\
v(0)=v(T) \text { in } \bar{\Omega} .
\end{array}\right.
$$


Proposition 12 yields for every $L>0$ the existence of a constant $C(L)>0$ such that, if $u$ is a fixed point of $T_{l}$ with $0 \leqslant l \leqslant L$, then we have $\|u\|_{L^{\infty}\left(Q_{T}\right)} \leqslant C(L)$. Then, multiplying (17) (with $v=u$ ) by a positive solution of

$$
\left\{\begin{array}{l}
\left(-\tilde{v}_{1}\right)_{t}-\Delta \tilde{v}_{1}=\lambda_{1}(m) m(t) \tilde{v}_{1} \text { in } Q_{T}, \\
\tilde{v}_{1}=0 \text { on } S_{T}, \\
\tilde{v}_{1}(0)=v_{1}(T) \text { in } \bar{\Omega}
\end{array}\right.
$$

(observe that (18) is the adjoint problem of (2) when $\mathbf{L}=(\partial / \partial t)-\Delta$, hence $\tilde{v}_{1}$ exists), we easily see that, for some $\bar{L} \geqslant \lambda_{1}(m), T_{\bar{L}}$ has no fixed points. Moreover if $u$ is a fixed point of $T_{l}$ and $\|u\|_{L^{\infty}\left(Q_{T}\right)} \leqslant \beta$, there is a constant $M_{\beta}$ verifying $\|u\|_{W^{1, q}\left(Q_{T}\right)} \leqslant M_{\beta}$, where $M$ depends only on $\|m\|_{L^{\infty}(0, T)}$, on $\beta$ and on $(\operatorname{meas}(\Omega))^{1 / q}$. Therefore for $l \in[0, \bar{L}], T_{l}$ has no fixed points on $\partial B_{M_{C(\bar{L})}}$, and the function $d\left(I-T_{l}, B_{M_{C(\bar{L}},}, 0\right)$ is well defined and constant. Hence, since $T_{0}=K_{1}$, we finally have $d\left(I-K_{1}, B_{M_{C(I)},}, 0\right)=0$. Finally, we use the degree's excision property and we find that $d\left(I-K_{1}, B_{M_{C(\bar{L})}}-\bar{B}_{\delta^{\prime}}, 0\right)=-1$. And this ends the proof.

Next we give the auxiliary results that will enable us to prove Theorems 6 and 7 . In every case the aim is to obtain $L^{\infty}$-estimates for the solutions of (4).

Proposition 14. Under assumptions (6)-(8) and if $1<\alpha<(N+1) /(N-1)$, there exists a positive constant $C$ such that, for any classical solution $u$ of (4), we have $\|u\|_{L^{\infty}\left(Q_{T}\right)} \leqslant C$.

Proof. By Proposition 9, $u$ is uniformly bounded in $L^{\alpha}\left(Q_{T}\right)$. Let us prove next the existence of $H^{1}\left(Q_{T}\right)$-estimates on $u$.

We multiply (4) by $u$ and $u_{t}$, we integrate by parts and we add the resulting equalities. Since $m$ satisfies (7)-(8) we have

$$
\begin{aligned}
\|u\|_{H^{1}\left(Q_{T}\right)}^{2} & =\iint_{Q_{T}}\left(m(t) u^{\alpha} u_{t}+m(t) u^{\alpha+1}\right) d t d x \\
& =\iint_{Q_{T}} u^{\alpha+1}\left(m(t)-\frac{m^{\prime}(t)}{\alpha+1}\right) d t d x \leqslant C \iint_{Q_{T}} u^{\alpha+1} d t d x .
\end{aligned}
$$

Since $\alpha<(N+1) /(N-1), \alpha+1<2+2 \alpha /(N+1)$. Then for all $\varepsilon>0$ there is a constant $C_{\varepsilon}>0$ such that

$$
\begin{aligned}
\iint_{Q_{T}} u^{\alpha+1} d t d x \leqslant & C_{\varepsilon}+\varepsilon\left(\iint_{Q_{T}} u^{2(N+1) /(N-1)} d t d x\right)^{(N-1) /(N+1)} \\
& \times\left(\iint_{Q_{T}} u^{\alpha} d t d x\right)^{2 /(N+1)}
\end{aligned}
$$

but $H^{1}\left(Q_{T}\right)$ is continuously embedded in $L^{2(N+1) /(N-1)}\left(Q_{T}\right)$ and $u$ is bounded in $L^{\alpha}$. Then choosing $\varepsilon$ small enough we finally have $\|u\|_{H^{1}\left(Q_{T}\right)} \leqslant C$.

The $H^{1}$-estimates yield an $L^{2}$-estimate for $u^{\alpha}$. We then end the proof by using the same boot-strap argument as in Proposition 12.

Let us now look for $L^{\infty}$-estimates for the solutions of (4) when we are under the assumptions of Theorem 7 . 
LEMMA 15. If $u$ is a classical solution of (4) and $m$ satisfies (7)-(8), the following equality holds:

$$
\begin{array}{r}
\iint_{Q_{T}}\left(\frac{2 N}{\alpha+1}-(N-2)\right) u^{\alpha+1} m(t) d t d x \\
=\iint_{Q_{T}}\left(\frac{m^{\prime}(t)}{\alpha+1} u^{\alpha+1}+u_{t}^{2}\right) \frac{|x|^{2}}{2} d t d x \\
\quad+\iint_{S_{T}}|\nabla u|^{2}(x \cdot n(x)) m(t) d t d x
\end{array}
$$

Remark. This lemma yields a kind of Pohozaev-identity (see [21]) for periodic solutions of the parabolic problem (4).

Proof of Lemma 15. Equation (19) is easily obtained multiplying (4) by ( $\sum_{i=1}^{N} u_{i} x_{i}$ $\left.-u_{t}|x|^{2} / 2\right)$ and integrating by parts.

COROllary 16. If $u$ is a solution of (4) and $m$ satisfies (7)-(8), the following inequality holds:

$$
\begin{aligned}
& \frac{1}{2} \iint_{Q_{T}}\left(R^{2}-|x|^{2}\right) m^{\prime}(t) \frac{u^{\alpha+1}}{\alpha+1} d t d x \\
& \quad+\iint_{Q_{T}}\left(\frac{2 N}{\alpha+1}-(N-2)\right) u^{\alpha+1} m(t) d t d x \leqslant C,
\end{aligned}
$$

where $C$ does not depend on $u$, and $R=\sup _{x \in \Omega}|x|$.

Proof. It suffices to notice that

$$
\iint_{Q_{T}} u_{t}^{2} d t d x=-\frac{1}{\alpha+1} \iint_{Q_{T}} m^{\prime}(t) u^{\alpha+1} d t d x
$$

Then we have

$$
\iint_{Q_{T}} \frac{|x|^{2}}{2} u_{t}^{2} d t d x \leqslant \frac{R^{2}}{2} \iint_{Q_{T}} u_{t}^{2} d t d x=-\frac{R^{2}}{2(\alpha+1)} \iint_{Q_{T}} m^{\prime}(t) u^{\alpha+1} d t d x
$$

We finally use Lemma 10 to bound the boundary integral in (19), and the proof is completed.

Proposition 17. If $m$ satisfies assumptions (6)-(9) and

$$
1<\alpha<(3 N+8) /(3 N-4),
$$

then all solutions of (4) are uniformly bounded in $L^{\infty}\left(Q_{T}\right)$.

Proof. This proof is divided into four steps. First, we obtain $H^{1}\left(Q_{T}\right)$-estimates for the solutions $u$ of (4). Then we deduce the uniform boundedness of $u$ in $L^{4}\left(0, T ; H_{0}^{1}(\Omega)\right)$. In the third step we observe that these estimates imply in fact an $L^{\infty}\left(0, T ; L^{p}(\Omega)\right)$-estimate, for $p<6 N /(3 N-4)$. Finally, we infer the $L^{\infty}\left(Q_{T}\right)$ estimates for $u$. Let us remark that in the third and fourth steps we follow some arguments used by T. Cazenave and P. L. Lions in [8]. 
First step: $u$ is bounded in $H^{1}\left(Q_{T}\right)$.

As we did in the proof of Proposition 14, we multiply (4) by $u$ and $u_{t}$, we integrate by parts and add the resulting equalities to get

$$
\|u\|_{H^{1}\left(Q_{T}\right)}^{2}=\iint_{Q_{T}}\left(m(t)-\frac{m^{\prime}(t)}{\alpha+1}\right) u^{\alpha+1} d t d x
$$

Now since $m$ satisfies (9), there is a constant $C>0$ and a point $\bar{x} \in \mathbf{R}^{N}$ that we choose as origin, such that we have

$$
m(t)-\frac{m^{\prime}(t)}{\alpha+1} \leqslant C\left[\left(\frac{2 N}{\alpha+1}-(N-2)\right) m(t)+\frac{R^{2}-|x|^{2}}{2(\alpha+1)} m^{\prime}(t)\right],
$$

where $R=\max _{x \in \bar{\Omega}}|x|$.

Finally we apply Corollary 16 to prove that all solutions of (4) are uniformly bounded in $H^{1}\left(Q_{T}\right)$ if $m$ satisfies (7)-(9).

Second step: $u$ is bounded in $L^{4}\left(0, T ; H_{0}^{1}(\Omega)\right)$.

For $t \in[0, T]$ we define the "action" function as follows:

$$
S(u(t))=\frac{1}{2} \int_{\Omega}|\nabla u(t)|^{2} d x-\frac{m(t)}{\alpha+1} \int_{\Omega} u(t)^{\alpha+1} d x .
$$

First, we verify that $S(u)$ is uniformly bounded in $L^{\infty}(0, T)$. Indeed, since $u$ is uniformly bounded in $H^{1}\left(Q_{T}\right)$ and $\|u\|_{L^{\alpha+1}}^{\alpha+1}\left(Q_{T}\right) \leqslant C\|\nabla u\|_{L^{2}\left(Q_{T}\right)}^{2}$, it is obvious that $S(u(\cdot))$ is bounded in $L^{1}(0, T)$. Moreover, for all $s, t \in[0, T]$ we have

$$
\begin{aligned}
S(u(t))-S(u(s))= & \frac{1}{2} \int_{s}^{t} \frac{\partial}{\partial t} \int_{\Omega}|\nabla u(\tau)|^{2} d x d \tau-\int_{s}^{t} \int_{\Omega} m^{\prime}(\tau) \frac{u^{\alpha+1}(\tau)}{\alpha+1} d \tau d x \\
& -\int_{s}^{t} \int_{\Omega} m(\tau) u^{\alpha}(\tau) d x d \tau
\end{aligned}
$$

and using (4) we find

$$
S(u(t))-S(u(s))=-\int_{s}^{t} \int_{\Omega}\left|u_{t}\right|^{2} d x d \tau-\int_{s}^{t} \int_{\Omega} m^{\prime}(\tau) \frac{u^{\alpha+1}(\tau)}{\alpha+1} d x d \tau,
$$

and then $|S(u(t))-S(u(s))| \leqslant C \forall s, t \in[0, T]$. Hence $S(u(\cdot))$ is bounded in $L^{\infty}(0, T)$ independently of $u$.

On the other hand, there exist a constant $C$ and an $s \in[0, T]$ such that $\|u(s)\|_{L^{2}(\Omega)},\left\|u_{t}\right\|_{L^{2}\left(Q_{T}\right)} \leqslant C$. Therefore $\|u\|_{L^{\infty}\left(0, T ; L^{2}(\Omega)\right)} \leqslant C$.

Finally, we multiply (4) by $u$ and integrate over $\Omega$. We find

$$
\int_{\Omega} u(t) u_{t}(t) d x+\int_{\Omega}|\nabla u(t)|^{2} d x=\int_{\Omega} m(t) u(t)^{\alpha+1} d x .
$$

But

$$
\begin{aligned}
\int_{\Omega} m(t) u(t)^{\alpha+1} d x & =\frac{\alpha+1}{2} \int_{\Omega}|\nabla u(t)|^{2} d x-S(u(t)) \\
& \geqslant \frac{\alpha+1}{2} \int_{\Omega}|\nabla u(t)|^{2} d x-C .
\end{aligned}
$$

Then using the above estimate we have $\|\nabla u(t)\|_{L^{2}(\Omega)}^{2} \leqslant C+C\left\|u_{t}\right\|_{L^{2}(\Omega)}$. Hence, $\|u\|_{L^{4}\left(0, T ; H_{0}^{1}(\Omega)\right)} \leqslant C$. 
Third step: $u$ is bounded in $L^{\infty}\left(0, T ; L^{p}(\Omega)\right)$ for all $p<6 N /(3 N-4)$.

This estimate is obtained by a direct application of the interpolation theory (see $\mathrm{J}$. Bergh and J. Löfstrom [5]). Actually if $u$ is bounded in $L^{4}\left(0, T ; L^{2 N /(N-2)}(\Omega)\right)$ and $u_{t}$ is bounded in $L^{2}\left(Q_{T}\right)$, then $u$ is bounded in $L^{\infty}\left(0, T ; L^{6 N /(3 N-4), 3}(\Omega)\right)$, and subsequently in all the $L^{\infty}\left(0, T ; L^{p}(\Omega)\right)$ for $p<6 N /(3 N-4)$, where $L^{\epsilon N /(3 N-4), 3}(\Omega)$ is a Lorentz space (for the definition and properties of it see [5]). In [8] we can find a simple proof of a slightly weaker version of it, which is sufficient for us.

Fourth step: $u$ is bounded in $L^{\infty}\left(Q_{T}\right)$.

The $L^{\infty}$-estimate follows from the third step by a straightforward application of a theorem due to O. A. Ladyzenskaja, V. A. Solonnikov and N. N. Ural'ceva [15]. Indeed since we have $u_{t}-\Delta u=m(t) u^{\alpha-1} u$ and, by the third step, $u$ is bounded in $L^{\infty}\left(0, T ; L^{p}(\Omega)\right), \quad p<6 N /(3 N-4)$, the function $\left(m(t) u^{\alpha-1}\right)$ is bounded in $L^{\infty}\left(0, T ; L^{q}(\Omega)\right)$, for $q<6 N /(3 N-4)(\alpha-1)$. Then, since $N / 2 q<1$ we deduce from Theorem 8.1 of $\left[15\right.$, p. 192] that $u \in L^{\infty}\left(Q_{T}\right)$.

Proof of Theorems 6 AND 7. Once we have obtained the $L^{\infty}$-estimates for all the solutions of (4), the proof is completed in the same way as in the case of Theorem 4.

IV. The general case (A). In $\S \S I I$ and III we have considered (4) in the particular case where $f(t, x, s)=m(t) s^{\alpha}$. We will state here the corresponding results when $f$ is of the form $f(t, x, s)=m(t) g(s)$, with $g$ superlinear.

Since the proofs of the results are explicitly written for the simpler case $g(s)=s^{\alpha}$, we will skip the proofs in the general case: the ideas are quite the same as those of $\S I I I$.

First we give the general assumptions that $g$ will satisfy

$$
\begin{gathered}
g(0)=0 \quad \text { and } \quad g \in W_{\mathrm{loc}}^{1, \infty}\left(\mathbf{R}^{+}\right), \\
\underset{s \rightarrow+\infty}{\lim } \frac{g(s)}{s}>\lambda_{1}(m), \\
\varlimsup_{s \rightarrow 0^{+}} \frac{g(s)}{s}<\lambda_{1}(m),
\end{gathered}
$$

if $\Omega$ is not convex, $\left(g(s) s^{-(N+2) /(N-2)}\right)$ is nonincreasing for $s \geqslant 0$. (If $N=2$ no condition is needed.)

Obviously all these assumptions are satisfied by $g(s)=s^{\alpha}$. We can now state the results we will prove:

THEOREM 4'. Assume (5)-(6) and (22)-(24). Then, if

$$
\varlimsup_{s \rightarrow+\infty} \frac{g(s)}{s^{\sigma}}=0 \quad \text { for some } \sigma<\frac{N+2}{N},
$$

(4) has at least one solution in $C^{2+\mu, 1+\mu / 2}\left(Q_{T}\right)$ for $0<\mu<1$.

REMARK. Theorem $4^{\prime}$ is still valid when we consider in (4) any elliptic operator $L$, instead of $(-\Delta)$. 
TheOREM 6'. Assume that $m$ and $g$ satisfy (6)-(8) and (22)-(25) and

$$
\varlimsup_{s \rightarrow+\infty} \frac{g(s)}{s^{\sigma}}=0 \quad \text { for some } \sigma<\frac{N+1}{N-1},
$$

$$
\begin{aligned}
& G(s) s^{-\delta} \text { is nondecreasing for } s \text { large and for some } \delta>0, \text { where } \\
& G(s)=\int_{0}^{s} g(r) d r .
\end{aligned}
$$

Then (4) has at least one solution in $C^{2+\mu, 1+(\mu / 2)}\left(Q_{T}\right)$ for $0<\mu<1$.

THEOREM $7^{\prime}$. Let us suppose that $m$ and $g$ satisfy (6)-(8), (22)-(25) and

$$
\begin{gathered}
\varlimsup_{s \rightarrow+\infty} \frac{g(s)}{s^{\sigma}}=0 \text { for some } \sigma<\frac{3 N+8}{3 N-4}, \\
\varlimsup_{s \rightarrow+\infty} \frac{s g(s)-\theta G(s)}{s^{2}(g(s))^{2 / N+1}} \leqslant 0 \quad \text { for some } \theta \in\left(1, \frac{2 N}{N-2}\right), \\
\sup _{0 \leqslant t \leqslant T} \frac{\left(m^{\prime}(t)\right)^{-}}{m(t)}<\frac{4 N-2 \theta(N-2)}{R(\Omega)}
\end{gathered}
$$

( for the definition of $R(\Omega)$, see $(9)$ ).

Then there is at least one solution of (4) in $C^{2+\mu, 1+\mu / 2}\left(Q_{T}\right)$ for $0<\mu<1$.

We point out again that the proofs of all these results are exactly the same as the ones we did. The assumptions made here are only the properties of power functions that we used in the preceding two sections.

V. The case (B). We consider here an eigenvalue problem related to (4):

$$
\left\{\begin{array}{l}
u_{t}-\Delta u=\lambda f(t, x, u)+h(t, x) \text { in } Q_{T} \\
u=0 \text { on } S_{T} ; \quad u>0 \text { in } Q_{T} \\
u(0)=u(T) \text { in } \bar{\Omega}
\end{array}\right.
$$

where $f$ and $g$ satisfy the following assumptions:

$$
\begin{gathered}
h \in C\left(\bar{Q}_{T} ; \mathbf{R}^{+}\right), \quad f \in C\left(\bar{Q}_{T} \times \mathbf{R}^{+} ; \mathbf{R}^{+}\right) \text {and } f(t, x, \cdot) \in C^{1}\left(\mathbf{R}^{+}\right), \\
f(t, x, 0) \not \equiv 0 \quad \text { if } h \equiv 0, \\
\inf _{s \geqslant 0} f_{s}^{\prime}(t, x, s)>0 \quad \text { uniformly in }(t, x) \in \bar{Q}_{T} .
\end{gathered}
$$

REMARK 18. We may take in (32) any elliptic operator $L$, and not necessarily $(-\Delta)$.

If we consider (32) with $\lambda=0$, this problem has a unique solution $u_{0} \in$ $C^{2+\alpha, 1+\alpha / 2}\left(Q_{T}\right)$ for $\alpha \in(0,1)$. (Note that if $h \equiv 0, u_{0} \equiv 0$, and that if $h \geqslant 0$, $h \not \equiv 0$, then $u_{0}>0$ in $Q_{T}$.) 
Let us now state the most general result which holds in the above situation:

Proposition 19. Let $S=\left\{\left(0, u_{0}\right)\right\} \cup\{(\lambda, u):(\lambda, u)$ is a solution of (32) $\}$, and define $S_{0}$ as the connected component of $S$ which contains $\left(0, u_{0}\right)$. Then $S_{0}$ is unbounded in $C\left(\bar{Q}_{T}\right) \times[0,+\infty)$. Moreover, there exist two constants $\underline{\lambda}, \bar{\lambda} \in(0,+\infty)$, $\underline{\lambda} \leqslant \bar{\lambda}$, such that:

(i) Problem (32) has no solution for $\lambda>\bar{\lambda}$.

(ii) $S_{0}$ contains a continuous curve $\mathscr{A}=\{(\lambda, u(\lambda)), 0 \leqslant \lambda<\underline{\lambda}\}\left(u_{0}=u(0)\right)$ such that, for every $\lambda \in[0, \underline{\lambda}), u(\lambda)$ is a minimal solution of (32).

(iii) If $f(t, x, \cdot)$ is convex in $\mathbf{R}^{+}$, then $(d / d \lambda) u(\lambda) \geqslant 0$ for all $\lambda$ in $[0, \underline{\lambda})$.

REMARK 20. The similar result for the elliptic problem is well known. See, for example, M. G. Crandall and P. H. Rabinowitz [9], F. Mignot and J. P. Puel [19], D. G. de Figueiredo, P. L. Lions and R. Nussbaum [10] and P. L. Lions [18].

SKETCH OF THE PROOF. This proof closely follows the corresponding one of the elliptic case (see [9 or 18]). We will thus only give its general features.

First, we show that there is a branch $\mathscr{A}$ of solutions of (32) which goes through $\left(0, u_{0}\right)$ by application of the implicit function theorem. Since $u_{0}$ is the unique solution of (32) for $\lambda=0$, it readily follows that $S_{0}$ is unbounded by means of a topological degree argument.

Next, if $(\lambda, u)$ is a solution of (32), $\lambda$ must be less than or equal to $\lambda_{1}(f(t, x, u(t, x)) / u(t, x))$ (equal in the case $h \equiv 0$ ) for all $(t, x) \in Q_{T}$.

Thus, since $f(t, x, 0) \geqslant 0$ and $f$ satisfies (33)-(34), there is a constant $C>0$ such that

$$
\lambda_{1}\left(\frac{f(t, x, u(t, x))}{u(t, x)}\right) \leqslant \lambda_{1}(C) .
$$

This proves the existence of $\bar{\lambda}$.

On the other hand, if we denote by $u(\lambda)$ the solutions of (32) which are in the branch $\mathscr{A}$, we see that we are again able to use the implicit function theorem at $(\lambda, u(\lambda))$ as far as $\lambda \leqslant \lambda_{1}\left(f_{z}^{\prime}(t, x, u(\lambda))\right)$. Indeed, the implicit function theorem can be applied at $(\lambda, u(\lambda))$ if and only if the operator $v \rightarrow v_{t}-\Delta v-\lambda f_{z}^{\prime}(t, x, u(\lambda)) v$ is invertible as a map from $C^{2+\mu, 1+\mu / 2}\left(\bar{Q}_{T}\right)$ into $C^{\alpha}\left(\bar{Q}_{T}\right)$. Thus, we call $\underline{\lambda}$ the supremum of the $\lambda$ for which this is true.

Next we consider what happens at $\lambda=\underline{\lambda}$, that is, we try to know under which assumptions there exists a solution of $(32), \underline{u}$, for $\lambda=\underline{\lambda}$, and what happens to $S_{0}$ beyond $(\underline{\lambda}, \underline{u})$.

THEOREM 21. Under the assumptions of Proposition 19, there exists a solution of (32) for $\lambda=\underline{\lambda}, \underline{u}=\lim _{\lambda \lambda_{\lambda}} u(\lambda)$ if $\mathscr{A}=\{(\lambda, u(\lambda)) \mid 0 \leqslant \lambda \leqslant \underline{\lambda}\}$ is bounded in $\mathbf{R}^{+} \times C\left(\bar{Q}_{T}\right)$. Moreover, $(\underline{\lambda}, \underline{u})$ is a turning point for $S_{0}$, that is, $\mathscr{A}$ can be continued beyond $(\underline{\lambda}, \underline{u})$.

REMARK 22. If $\mathscr{A}$ is bounded, the above theorem shows that $\underline{\lambda}=\lambda_{1}\left(f_{z}^{\prime}(t, x, \underline{u})\right)$. Otherwise $\underline{\lambda}$ would not be maximal with respect to the existence of $\mathscr{A}$. 
REMARK 23. The proof of Theorem 21 is again almost the same as in the elliptic case, and we will skip it.

Finally, we will state a result which follows from the combination of results of types (A) and (B). It gives a more precise description of the solution-set of (32) when $h \equiv 0$ and $f$ is of the form $m(t) g(u)$, as in (4).

COROLlary 24. Assume that $m$ and $g$ satisfy the conditions of either Theorems 4, 6 or 7. In addition, we suppose

$$
\begin{gathered}
g \in C^{1}\left(\mathbf{R}^{+}\right), \quad g(0)>0 \text { and } \inf _{s \geqslant 0} g^{\prime}(s)>0, \\
\underset{s \rightarrow+\infty}{\frac{\lim }{s}} \frac{g(s)}{s}=+\infty .
\end{gathered}
$$

Then, if we take $f(t, x, z)=m(t) g(z)$ and $h \equiv 0$, Proposition 19 and Theorem 20 are satisfied. Moreover, $\underline{\lambda}=\bar{\lambda}$ and for all $\lambda \in(0, \underline{\lambda})$ there exist at least two solutions of (32) in $S_{0}$. If $g$ is strictly convex in $\mathbf{R}^{+}$, there is a unique solution of (32) for $\lambda=\underline{\lambda}$.

Sketch OF THE PROOF. That Proposition 19 and Theorem 20 apply to this situation is obvious, since all the conditions needed are satisfied.

Let us then prove the last statement of the corollary. It is done quite similarly as in the elliptic case (see [10]).

We denote by $\phi$ the inverse of $((\partial / \partial t)-\Delta)$ with Dirichlet boundary conditions and periodicity in $t$, with period $T$. Then, it is obvious that $u$ is a solution of (32) if and only if $u=\lambda \phi(u)$. Moreover, it is easy to see that $\phi$ is a compact operator from $C^{\alpha}\left(\bar{Q}_{T}\right)$ into itself $(0<\alpha<1)$.

On the other hand, we notice that since $g$ satisfies condition (37), for all $\varepsilon>0$, there exists a positive constant $M_{\varepsilon}$ such that

$$
u=\lambda \phi(u) \text { and } \varepsilon \leqslant \lambda \leqslant \bar{\lambda} \quad \text { imply }\|u\|_{L^{\infty}\left(Q_{T}\right)}<M_{\varepsilon} .
$$

Indeed, it suffices to apply the boundedness results obtained in §III.

Then, fixing $\varepsilon>0$ and denoting by $S_{\varepsilon}$ the set of the solutions of (32) for $\lambda \geqslant \varepsilon$, we can find an open bounded set of $(\varepsilon / 2,+\infty) \times C\left(\bar{Q}_{T}\right), W$, such that $\lambda \phi$ has no fixed points in $\partial W$, for $\varepsilon \leqslant \lambda \leqslant \bar{\lambda}$. We finish the proof by doing a simple topological degree argument. Indeed, define $W_{\lambda}=\left\{u \in C\left(\bar{Q}_{T}\right):(\lambda, u) \in W\right\}$. It is obvious that the degree $d\left(I-\lambda \phi, W_{\lambda}, 0\right)$ is constant for $\varepsilon \leqslant \lambda \leqslant \bar{\lambda}+1$, from the choice of $W$. Thus since $(\bar{\lambda}+1) \phi$ has no fixed points, it follows that $d\left(I-\lambda \phi, W_{\lambda}, 0\right)=0$ for all $\varepsilon \leqslant \lambda \leqslant \bar{\lambda}$.

From the method used to prove the existence of $\mathscr{A}$, it is easy to see that, for any $\lambda \in(0, \bar{\lambda})$, there exists an open bounded set $\mathcal{O}_{\lambda}$ such that $u(\lambda) \in \mathcal{O}_{\lambda}$ and $\lambda_{\phi}$ has no fixed point in $\overline{\mathcal{O}}_{\lambda}-\mathcal{O}_{\lambda}$. Thus, if we assume that for a $\lambda$ in $(0, \bar{\lambda}), \lambda \phi$ has only a fixed point $u(\lambda)$, it appears that $d\left(I-\lambda \phi, W_{\lambda}, 0\right)=d\left(I-\lambda \phi, \mathcal{O}_{\lambda}, 0\right)=+1$ or -1 . This contradiction shows the existence of a second solution of $(32)$ in $(0, \underline{\lambda})$. 
Let us suppose finally that, $g$ being strictly convex, there are two solutions of (32) for $\lambda=\underline{\lambda}, \underline{u}$ and $v$. Then, we have

$$
\left\{\begin{array}{l}
\underline{u}_{t}-\Delta \underline{u}=\underline{\lambda} g(\underline{u}) m(t) \quad \text { in } Q_{T}, \\
v_{t}-\Delta v=\underline{\lambda} g(v) m(t) \quad \text { in } Q_{T}, \\
\underline{u} / S_{T}=v / S_{T}=0 \\
\underline{u}, v>0 \quad \text { in } Q_{T}, \\
\underline{u}(0)=\underline{u}(T) \quad \text { and } \quad v(0)=v(T) \quad \text { in } \bar{\Omega} .
\end{array}\right.
$$

If we define $w=v-\underline{u}$, we find, for $w$,

$$
\left\{\begin{array}{l}
w_{t}-\Delta w=\underline{\lambda} l(t, x) m(t) w \quad \text { in } Q_{T}, \\
w(0)=w(T) \quad \text { in } \bar{\Omega}, \\
w=0 \quad \text { on } S_{T} ; \quad w \geqslant 0 \quad \text { in } \bar{Q}_{T}
\end{array}\right.
$$

with $l(t, x)>g^{\prime}(\underline{u}(t, x))$ for all $(t, x) \in Q_{T}$. Indeed, $\underline{u}$ must be the minimal solution and $g$ is strictly convex in $\mathbf{R}^{+}$.

Finally, we multiply (38) by a positive solution of

$$
\left\{\begin{array}{l}
-u_{t}-\Delta u=\underline{\lambda} g^{\prime}(\underline{u}) m(t) u \text { in } Q_{T}, \\
u=0 \text { on } S_{T}, \\
u(0)=u(T) \text { in } \bar{\Omega}
\end{array}\right.
$$

(see Remark 22 and notice that $-(\partial / \partial t)-\Delta$ is the adjoint operator of $(\partial / \partial t)-\Delta$, when we consider Dirichlet boundary conditions and periodicity) and we see that $w$ must be equal to 0 everywhere.

\section{REFERENCES}

1. H. Amann, Periodic solutions of semilinear parabolic equations, Nonlinear Analysis (Cesari-Kannan-Weinberger, eds.), Academic Press, New York, 1978, pp. 1-29.

2. Invariant sets and existence theorems for semilinear parabolic and elliptic systems, J. Math. Anal. Appl. 65 (1978), 432-467.

3. D. G. Aronson, On the Green's function for second order parabolic differential equations with discontinuous coefficients, Bull. Amer. Math. Soc. 69 (1963), 841-847.

4. A. Beltramo and P. Hess, On the principal eigenvalue of a periodic-parabolic operator, Preprint.

5. J. Bergh and J. Löfstrom, Interpolation spaces, Springer-Verlag, Berlin and New York, 1976.

6. H. Brézis and L. Nirenberg, Characterization of the ranges of some nonlinear operators, Ann. Sci. École Norm. Sup. (4) 5 (1978), 225-326.

7. A. Castro and A. C. Lazer, Results on periodic solutions of parabolic equations suggested by elliptic theory, Boll. Un. Mat. Ital. B(6) 1 (1982), 1089-1104.

8. T. Cazenave and P. L. Lions, Solutions globales d'équations de la chaleur semilinéaires, Preprint.

9. M. G. Crandall and P. H. Rabinowitz, Some continuation and variational methods for positive solutions of nonlinear elliptic eigenvalue problems, Arch. Rational Mech. Anal. 58 (1975), 207-218.

10. D. G. de Figueiredo, P. L. Lions and R. D. Nussbaum, A priori estimates and existence of positive solutions of semilinear elliptic equations, J. Math. Pures Appl. 61 (1982), 41-63.

11. B. Gidas, W.-M Ni and L. Nirenberg, Symmetry and related properties via the maximum principle, Comm. Math. Phys. 68 (1979), 209-243.

12. B. Gidas and J. Spruck, A priori bounds for positive solutions of non-linear elliptic equations, Comm. Partial Differential Equations 6 (1981), 883-901.

13. P. Hess, On positive solutions of semilinear periodic-parabolic problems, Proc. Conference on Operator-Semigroups and Applications, June 1983 (to appear). 
14. J. S. Kolesov, A test for the existence of periodic solutions of parabolic equations, Soviet Math. Dokl. 7 (1966), 1318-1320.

15. O. A. Ladyzenskaja, V. A. Solonnikov and N. N. Ural'ceva, Linear and quasi-linear equations of parabolic type, Transl. Math. Monographs, vol. 23, Amer. Math. Soc., Providence, R. I., 1968.

16. A. C. Lazer, Some remarks on periodic solutions of parabolic differential equations, Dynamical Systems. II (Bednarek-Cesari, eds.), Academic Press, New York, 1982, pp. 227-246.

17. J. Leray and J. Schauder, Topologie et équations fonctionnelles, Ann. Sci. École Norm. Sup. 51 (1934), 45-78.

18. P. L. Lions, On the existence of positive solutions in semilinear elliptic equations, MRC Technical Report \#2209, University of Wisconsin, Madison, 1980.

19. F. Mignot and J. P. Puel, Sur une classe de problèmes non linéaires avec non linéarité positive, croissante, convexe, Comm. Partial Differential Equations 5 (1980), 791-836.

20. L. Nirenberg, Topics in nonlinear functional analysis, N. Y. U., Courant Inst. Lecture Notes, New York, 1974.

21. S. I. Pohozaev, Eigenfunctions of the equation $\Delta u+\lambda f(u)=0$, Soviet Math. Dokl. 5 (1965), $1408-1411$.

Laboratoire d’Analyse Numerique, t. 55-65, Université Pierre et Marie Curie, 4 Place Jussieu, 75230 PARIS CedeX 05, France 